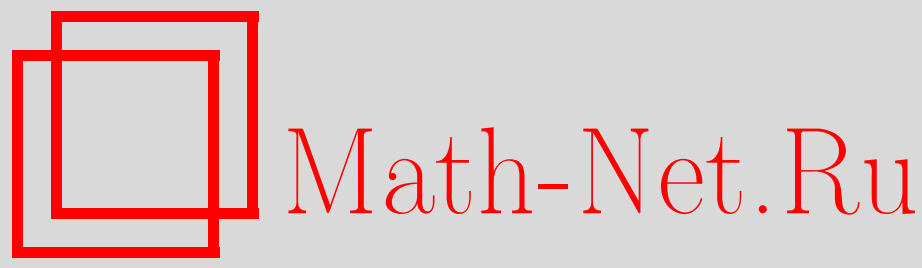

К. С. Карпов, Ю. М. Письмак, Алгебраический расчет резольвенты обобщенного квантового осциллятора в пространстве размерности $D, T M \Phi, 2015$, том 185, номер 1, 109-117

DOI: https://doi.org/10.4213/tmf8923

Использование Общероссийского математического портала Math-Net.Ru подразумевает, что вы прочитали и согласны с пользовательским соглашением http://www . mathnet.ru/rus/agreement

Параметры загрузки:

IP : 54.224 .60 .19

26 апреля 2023 г., 14:49:09

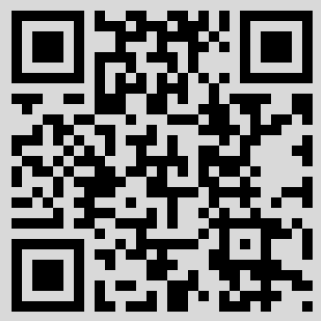




\title{
ФИЗИКА
}

Том 185, № 1

октябрь, 2015

(C) 2015 г. $\quad$ К. С. Карпов ${ }^{* \dagger}$, Ю. М. Письмак ${ }^{*}$

\section{АЛГЕБРАИЧЕСКИЙ РАСЧЕТ РЕЗОЛЬВЕНТЫ ОБОБЩЕННОГО КВАНТОВОГО ОСЦИЛЛЯТОРА В ПРОСТРАНСТВЕ РАЗМЕРНОСТИ $D$}

\begin{abstract}
Для изотропных моделей квантовой механики рассматривается формализм, основанный на применении алгебры $s l(2)$ вместо обычной алгебры Гейзенберга. В качестве ее образующих используются операторы квадрата импульса $p^{2}$, координаты $q^{2}$, а также оператор дилатации $H=i(p q+q p)$. Это дает возможность проводить расчеты, рассматривая размерность пространства $D$ в качестве произвольного, не обязательно целочисленного параметра. Для обобщенного гармонического осциллятора с гамильтонианом вида $\mathcal{H}(a, b, c)=a p^{2}+b q^{2}+c H$ и произвольного $D$ получены интегральные представления для резольвенты и ее следа. Изучены их аналитические свойства при различных значениях параметров модели.
\end{abstract}

Ключевые слова: обобщенный квантовый осциллятор, алгебра $s l(2)$, изотропные модели квантовой механики, резольвента, спектральное разложение.

DOI: $10.4213 / \operatorname{tmf} 8923$

\section{1. ВВЕДЕНИЕ}

В современной квантовой теории поля теоретические результаты, как правило, требуют большого объема вычислений кратных интегралов, представленных в виде диаграмм Фейнмана. При этом из-за возрастания сложности рассматриваемых задач приходится учитывать все более высокие порядки теории возмущений по константе связи и по количеству петель. Количество диаграмм с ростом порядка растет. Точность используемых для их расчетов численных методов падает, и они оказываются неэффективными. Вследствие этого становятся все более актуальными разработки аналитических методов вычисления многопетлевых диаграмм. Они проводятся весьма активно. В них используются различные алгебраические структуры [1], [2] и геометрические подходы [3], а также методы, основанные на аналитическом продолжении вкладов диаграмм по размерности пространства. В последнее

${ }^{*}$ Санкт-Петербургский государственный университет, Санкт-Петербург, Россия.

E-mail: karpovk@inbox.ru, ypismak@yahoo.com

${ }^{\dagger}$ Всероссийский научно-исследовательский институт метрологии им. Д. И. Менделеева, Санкт-Петербург, Россия 
время получен ряд важных результатов для диаграмм, используемых в расчетах $\varepsilon$-разложения критических индексов [4], [5] и при построении лестничных приближений [6].

Эффективные методы аналитических вычислений фейнмановских интегралов возникли в процессе построения $1 / n$-разложения для теории $\lambda\left(\phi^{2}\right)^{2}$ [7]. При этом использовались метод интегрирования по частям [8] и метод, основанный на соотношении "треугольник-звезда" (соотношение уникальности) [9]. В работе [10] было замечено, что это соотношение представляет собой вариант уравнения Янга-Бакстера.

В работе Исаева [11] был предложен новый алгебраический метод аналитического вычисления безмассовых и регуляризованных по размерности фейнмановских интегралов. Он основан на использовании структуры алгебры $s l(2)$, порождаемой операторами $\hat{p}^{2}, \hat{q}^{2}$ и $\widehat{H}$, а также оператора инверсии. Преимущество метода заключается в том, что в его рамках расчеты диаграмм Фейнмана проводятся алгебраически без громоздких процедур интегрирования. Это значительно упрощает все вычисления. В качестве примера в работе [11] приведен расчет лестничных диаграмм в теории скалярного безмассового поля $\phi$ с взаимодействием $\phi^{3}$. В этой работе показано также, что расчет некоторых классов диаграмм Фейнмана сводится к вычислению матричных элементов квантово-механических операторов. Для расчета лестничных диаграмм в модели $\phi^{3}$ оказывается достаточно найти оператор, обратный к гамильтониану в $D$-мерной конформной квантовой механике.

В настоящей работе мы используем алгебраический подход, предложенный в [11] для вычисления резольвенты $D$-мерного изотропного квантового осциллятора. Аналитический вид резольвенты в одномерном случае был получен в [12]. Трехмерный случай представлен в работе [13]. Наконец, обобщение этих результатов для произвольного конечномерного пространства получено в работе [14] на основе рекуррентных соотношений по числу пространственных измерений. В наших расчетах, основанных на коммутационных соотношениях алгебры $s l(2)$ для изотропных операторов квадратов импульса $p^{2}$, координаты $q^{2}$ и дилатации $H$, размерность пространства $D$ является параметром, который так же, как в методе размерной регуляризации квантовой теории поля, может быть выбран любым комплексным числом.

Для оператора Гамильтона обобщенного изотропного осциллятора, который является линейной комбинацией операторов $p^{2}, q^{2}$ и $H$, полученный нами результат для резольвенты зависит от трех входящих в гамильтониан параметров и размерности пространства $D$. Рассматривая резольвенту как функцию этих параметров, мы проводим анализ ее аналитических свойств.

\section{2. АЛГЕБРАИЧЕСКИЙ ПОДХОД В КВАНТОВОЙ МЕХАНИКЕ}

2.1. Алгебра Гейзенберга. Алгебра Гейзенберга лежит в основе операторной формулировки квантовой механики. Ее порождающими элементами являются операторы импульса и координаты. В $D$-мерном евклидовом пространстве $\mathbb{R}^{D}$

$$
\left[\hat{q}_{j}, \hat{p}_{k}\right]=i \delta_{j k}, \quad j, k=1, \ldots, D
$$

где $\hat{q}_{i}$ - оператор координаты, $\hat{p}_{i}$ - оператор импульса. Предполагается, что их собственные векторы состояний $|x\rangle,|k\rangle$ :

$$
\hat{q}_{i}|x\rangle=x_{i}|x\rangle, \quad \hat{p}_{i}|k\rangle=k_{i}|k\rangle,
$$


удовлетворяют нормировочным условиям и условию полноты:

$$
\begin{gathered}
\langle x \mid y\rangle=\delta^{D}(x-y), \quad\langle k \mid l\rangle=\delta^{D}(k-l), \quad\langle x \mid k\rangle=\frac{1}{(2 \pi)^{D / 2}} e^{i k_{j} x_{j}}, \\
\int d^{D} x|x\rangle\left\langle x\left|=\int d^{D} k\right| k\right\rangle\langle k|=1, \\
|k\rangle=\int|x\rangle\langle x \mid k\rangle d^{D} x=\frac{1}{(2 \pi)^{D / 2}} \int|x\rangle e^{i k x} d^{D} x .
\end{gathered}
$$

Динамика квантово-механической системы определяется оператором Гамильтона $\mathcal{H}=\mathcal{H}(\hat{p}, \hat{q})$, и задачи описания ее поведения сводятся к вычислению оператора эволюции $U(t)=e^{-i t \mathcal{H}}$. Его матричные элементы определяют амплитуды перехода. Для нас представляет интерес случай гамильтониана, инвариантного относительно поворотов в $D$-мерном пространстве.

2.2. Операторный формализм для изотропных моделей. Из коммутационных соотношений (1) следует, что инвариантный относительно вращений в $D$-мерном пространстве оператор $Q(\hat{p}, \hat{q})$ (в дальнейшем мы будем называть такие операторы изотропными) может быть записан в виде степенного ряда

$$
Q(\hat{p}, \hat{q})=\sum_{n_{1}, n_{2}, n_{3}=0}^{\infty} Q_{n_{1}, n_{2}, n_{3}} p^{2 n_{1}} q^{2 n_{2}} H^{2 n_{3}},
$$

где мы использовали обозначения Исаева [11] для операторов $p^{2} \equiv \hat{p}^{2}, q^{2} \equiv \hat{q}^{2}$, $H \equiv i(\hat{p} \hat{q}+\hat{q} \hat{p}) / 2$. Форму записи (2) операторов мы будем называть нормально упорядоченной.

2.3. Матричные элементы стандартных операторов. Операторы $e^{\alpha p^{2}}, e^{\beta q^{2}}$, $e^{\gamma H}$ мы будем называть стандартными. Приведем их матричные элементы, а также результаты действия на элементы базиса:

$$
\begin{gathered}
\langle x| e^{\beta q^{2}}=\langle x| e^{\beta x^{2}}, \quad\langle x| e^{t H}=\left\langle e^{t} x\right| e^{t D / 2}, \\
\left\langle x\left|e^{\alpha p^{2}}\right| y\right\rangle=(-4 \pi \alpha)^{-D / 2} e^{(x-y)^{2} / 4 \alpha} .
\end{gathered}
$$

2.4. Алгебра $\operatorname{sl}(2)$ и коммутационные соотношения изотропных операторов. Записывая операторы $p^{2}, q^{2}, H$ как квадратичные комбинации компонент операторов импульса и координаты $\hat{p}, \hat{q}$, нетрудно убедиться, используя коммутационные соотношения (1), что операторы $H, q^{2}, p^{2}$ образуют алгебраическую структуру $\operatorname{sl}(2)[11]$ :

$$
\left[q^{2}, p^{2}\right]=4 H, \quad\left[H, q^{2}\right]=2 q^{2}, \quad\left[H, p^{2}\right]=-2 p^{2} .
$$

Используя (5), можно вычислить коммутационные соотношения стандартных операторов с $H, q^{2}, p^{2}$. Введем обозначение

$$
A(\alpha, X, Y)=e^{\alpha X} Y e^{-\alpha X},
$$

где $\alpha$ - числовой параметр, $X, Y$ - два некоммутирующих оператора. Оператор $A(\alpha, X, Y)$ удовлетворяет соотношениям

$$
\frac{\partial}{\partial \alpha} A(\alpha, X, Y)=e^{\alpha X}[X, Y] e^{-\alpha X}, \quad A(0, X, Y)=Y .
$$


Таким образом, в силу (5) операторы $A(\alpha, X, Y)$ для $X, Y=H, p^{2}, q^{2}$ являются решениями линейных дифференциальных уравнений (6), что можно использовать для вывода соотношений

$$
\begin{aligned}
e^{\alpha H} p^{2} e^{-\alpha H} & =p^{2} e^{-2 \alpha}, & e^{\alpha H} q^{2} e^{-\alpha H} & =q^{2} e^{2 \alpha}, \\
e^{\alpha p^{2}} q^{2} e^{-\alpha p^{2}} & =q^{2}-4 \alpha\left(H+\alpha p^{2}\right), & e^{\alpha p^{2}} H e^{-\alpha p^{2}} & =H+2 \alpha p^{2}, \\
e^{\alpha q^{2}} p^{2} e^{-\alpha q^{2}} & =p^{2}+4 \alpha\left(H-\alpha q^{2}\right), & e^{\alpha q^{2}} H e^{-\alpha q^{2}} & =H-2 \alpha q^{2} .
\end{aligned}
$$

В работе Исаева [11] дается определение нецелой степени оператора на основе интегрального представления

$$
x^{-\omega}=\frac{1}{\Gamma(\omega)} \int_{0}^{\infty} e^{-\alpha x} \alpha^{\omega-1} d \alpha .
$$

Оно дает возможность, воспользовавшись (7), получить коммутационные соотношения

$$
\begin{gathered}
{\left[q^{2}, p^{2(\alpha+1)}\right]=4(\alpha+1)(H+\alpha) p^{2 \alpha},} \\
{\left[q^{2(\alpha+1)}, p^{2}\right]=4(\alpha+1)(H-\alpha) q^{2 \alpha},} \\
{\left[H q^{2 \alpha}, q^{2 \alpha}\right]=2 q^{2 \alpha} \alpha, \quad\left[H, p^{2 \alpha}\right]=-2 p^{2 \alpha} \alpha,}
\end{gathered}
$$

которые использовались в [11] и выполняются для любых значений показателя степени $\alpha$.

Рассмотрим теперь задачу вычисления матричных элементов резольвенты для оператора Гамильтона обобщенного гармонического осциллятора.

\section{3. ИЗотРопныЙ ОСцИЛлятОР}

Изотропный оператор $\mathcal{H}(a, b, c)=a p^{2}+b q^{2}+c H$ при $a>0, b>0, c=0$ является оператором Гамильтона гармонического осциллятора в $D$-мерном пространстве. Мы будем называть $\mathcal{H}(a, b, c)$ при произвольных параметрах $a, b, c$ гамильтонианом обобщенного изотропного осциллятора. Применяя приведенные выше результаты, вычислим резольвенту

$$
R(\lambda)=(\mathcal{H}-\lambda)^{-1}=\int_{0}^{\infty} e^{-t(\mathcal{H}-\lambda)} d t .
$$

Приведем оператор в подынтегральном выражении (9) к нормально упорядоченному виду. Мы получим нужную нам форму записи, если найдем $\alpha, \beta, \gamma$, для которых выполняется равенство

$$
e^{t\left(a p^{2}+b p^{2}+c H\right)}=e^{\alpha(a, b, c ; t) p^{2}} e^{\beta(a, b, c ; t) q^{2}} e^{\gamma(a, b, c ; t) H} .
$$

Тогда оператор резольвенты запишется в виде

$$
R(\lambda)=\int_{0}^{\infty} e^{\alpha(a, b, c ;-t) p^{2}} e^{\beta(a, b, c ;-t) q^{2}} e^{\gamma(a, b, c ;-t) H} e^{t \lambda} d t,
$$

и, воспользовавшись (3), (4), мы получаем для матричных элементов резольвенты $R(\lambda)$ и ее следа $\operatorname{Tr} R(\lambda)$ следующие выражения:

$$
\begin{aligned}
& R_{\lambda}(x, y)=\langle x|R(\lambda)| y\rangle=\int_{0}^{\infty} \frac{1}{\left(-4 \pi \alpha e^{\gamma}\right)^{D / 2}} \exp \left[\frac{\left(x-e^{-\gamma} y\right)^{2}}{4 \alpha}+\beta e^{-2 \gamma} y^{2}+t \lambda\right] d t \\
& \operatorname{Tr} R(\lambda)=\int R_{\lambda}(x, x) d^{D} x=\int_{0}^{\infty}\left(e^{\gamma}+(1+4 \alpha \beta) e^{-\gamma}-2\right)^{-D / 2} e^{\lambda t} d t
\end{aligned}
$$


Продифференцировав (10) по параметру $t$, получим

$$
\begin{aligned}
\left(a p^{2}\right. & \left.+b q^{2}+c H\right) e^{t\left(a p^{2}+b p^{2}+c H\right)}= \\
& =\frac{d \alpha}{d t} p^{2} e^{\alpha p^{2}} e^{\beta q^{2}} e^{\gamma H}+\frac{d \beta}{d t} e^{\alpha p^{2}} q^{2} e^{\beta q^{2}} e^{\gamma H}+\frac{d \gamma}{d t} e^{\alpha p^{2}} e^{\beta q^{2}} H e^{\gamma H} .
\end{aligned}
$$

С помощью соотношений (8) в этом равенстве множители $q^{2}$ и $H$ перед экспонентами можно переставить в крайнее левое положение и приравнять коэффициенты при одинаковых операторах. В результате для $\alpha, \beta, \gamma$ получается следующая система уравнений:

$$
\begin{aligned}
& a=\frac{\partial \alpha}{\partial t}-4 \frac{d \beta}{\partial t} \alpha^{2}+\frac{d \gamma}{\partial t}\left(2 \alpha+8 \alpha^{2} \beta\right), \\
& b=\frac{\partial \beta}{\partial t}-2 \beta \frac{\partial \gamma}{\partial t} \\
& c=-4 \alpha \frac{\partial \beta}{\partial t}+(1+8 \alpha \beta) \frac{\partial \gamma}{\partial t} .
\end{aligned}
$$

Функции $\alpha, \beta, \gamma$ удовлетворяют начальным условиям $\alpha=\beta=\gamma=0$ при $t=0$. Уравнения (13) имеют решения вида

$$
\begin{gathered}
\alpha=a \frac{\operatorname{sh}(f t)}{c \operatorname{sh}(f t)+f \operatorname{ch}(f t)}, \quad \beta=\frac{b}{f^{2}} \operatorname{sh}(f t)(c \operatorname{sh}(f t)+f \operatorname{ch}(f t)), \\
\gamma=\ln \frac{c \operatorname{sh}(f t)+f \operatorname{ch}(f t)}{f},
\end{gathered}
$$

где $f=\sqrt{4 a b+c^{2}}$.

3.1. Явный вид резольвенты и ее след. Подставляя $\alpha, \beta, \gamma$ из (14) в выражение для резольвенты (11), получим

$$
\begin{aligned}
R(x, y) & =\int_{0}^{\infty} e^{t \lambda}\left\langle x\left|e^{\alpha p^{2}} e^{\beta q^{2}} e^{\gamma H}\right| y\right\rangle d t= \\
& =\int_{0}^{\infty}\left(4 \pi \frac{a \operatorname{sh}(f t)}{f}\right)^{-D / 2} e^{F(x, y, a, c, f)+t \lambda} d t \\
F(x, y, a, c, f) & =\frac{c \operatorname{sh}(f t)\left(x^{2}-y^{2}\right)-f \operatorname{ch}(f t)\left(x^{2}+y^{2}\right)+2 f x y}{4 a \operatorname{sh}(f t)} .
\end{aligned}
$$

Отметим, что интеграл (15) может сходится только при $\operatorname{Re} \lambda<0$, и в дальнейшем мы всегда предполагаем, что это условие выполнено. Для тех значений параметров $a, c, f, D$, для которых интеграл расходится, выражение (15) для резольвенты понимается как аналитическое продолжение из той области их значений, в которой он существует. В силу (12), (14) имеем

$$
\operatorname{Tr} R(\lambda)=\int_{0}^{\infty}\left(2 \operatorname{sh}\left(\frac{f t}{2}\right)\right)^{-D} e^{t \lambda} d t .
$$

При $D=1$ мы получаем

$$
\operatorname{Tr} R(\lambda)=\int_{0}^{\infty} \sum_{n=0}^{\infty} e^{(\lambda-(2 n+1) f / 2) t} d t
$$


Отсюда легко находится спектр гамильтониана:

$$
\lambda_{n}=f\left(n+\frac{1}{2}\right)
$$

что совпадает со спектром, который получается стандартными методами с использованием операторов рождения и уничтожения.

3.2. Спектр и его вырождение. Рассмотрим след резольвенты для пространства размерности $D$. Воспользовавшись (16), можно показать, что при $f \neq 0$

$$
\operatorname{Tr} R(\lambda)=\int_{0}^{\infty} \sum_{n=0}^{\infty} C_{n}(D) e^{-(n+D / 2) f t} e^{t \lambda} d t
$$

и для спектра $\lambda_{n}$ резольвенты и числа соответствующих вырожденных состояний $C_{n}(D)$ получаются следующие выражения:

$$
\lambda_{n}=\frac{2 n+D}{2} f, \quad C_{n}(D)=\frac{\Gamma(D+n)}{\Gamma(D) \Gamma(n+1)} .
$$

Таким образом, при $f \neq 0$ спектр дискретный, и $C_{n}(D)$ является целым числом только при целом $D$.

3.3. Спектральное разложение резольвенты. Спектральное разложение для резольвенты записывается в виде

$$
R_{\lambda}(x, y)=\sum_{n=0}^{\infty} \frac{P_{n}(x, y)}{\lambda_{n}-\lambda}
$$

где $P_{n}(x, y)$ - проектор на соответствующее $\lambda_{n}$ собственное подпространство $R_{\lambda}(x, y)$, и при условии $f \neq 0$

$$
\begin{aligned}
P_{n}(x, y) & =\left.\frac{1}{n !} \frac{d^{n}}{d A^{n}}\left(\frac{f}{2 \pi a\left(1-A^{2}\right)}\right)^{D / 2} e^{Q(x, y, A)}\right|_{A=0}, \\
Q(x, y, A) & =\frac{c\left(1-A^{2}\right)\left(x^{2}-y^{2}\right)-f\left(1+A^{2}\right)\left(x^{2}+y^{2}\right)+4 A f x y}{4 a\left(1-A^{2}\right)} .
\end{aligned}
$$

Воспользовавшись формулой для гауссова интеграла в $D$-мерном пространстве

$$
\int e^{-x K x / 2+x y} d^{D} x=\left(\operatorname{det} \frac{K}{2 \pi}\right)^{-1 / 2} e^{y K^{-1} y / 2},
$$

нетрудно убедиться, что для проекторов при любом $D$ выполняется условие ортогональности:

$$
\int P_{m}(x, y) P_{n}(y, z) d^{D} y=\left.\frac{1}{m ! n !} \frac{d^{m+n} e^{Q(A B, x, z)}}{d A^{m} d B^{n}}\right|_{A=B=0}=P_{n}(x, z) \delta_{n m} .
$$

Можно также доказать, что

$$
\int_{-\infty}^{\infty} \sum_{n=0}^{\infty} P_{n}(x, y) e^{-K x^{2} / 2+J x} d^{D} x=e^{-K y^{2} / 2+J y}
$$


и, следовательно,

$$
\sum_{n=0}^{\infty} P_{n}(x, y)=\delta(x-y) .
$$

При целочисленных $D$ проектор $P_{n}(x, y)$ представляется в виде

$$
P_{n}(x, y)=\sum_{k=1}^{C_{n}(D)} \psi_{k}^{(n)}(x) \bar{\psi}_{k}^{(n)}(y),
$$

где $\psi_{k}^{(n)}(x), 1 \leqslant k \leqslant C_{n}(D),-$ собственные функции резольвенты

$$
R(x, y) \psi_{k}^{(n)}(x)=\frac{1}{\lambda_{n}-\lambda} \psi_{k}^{(n)}(x), \quad \int \psi_{k}^{(n)}(x) \bar{\psi}_{l}^{(m)}(x) d x=\delta_{n m} \delta_{k l},
$$

$C_{n}(D)$ - степень вырождения спектра (17).

3.4. Резольвента при различных значениях параметра $c$. Предполагая $a>0, b>0$, рассмотрим свойства резольвенты в двух случаях: при вещественном и мнимом параметре $c$.

3.4.1. Осциллятор без оператора дилатации $(c=0)$. Если $c=0$, оператор дилатации $H$ не содержится в $\mathcal{H}(a, b, 0)$, и $\mathcal{H}\left(1 /(2 m), m \omega^{2} / 2,0\right)$ является оператором Гамильтона обычного гармонического осциллятора. Для него из (15), (16) получаются следующие выражения для резольвенты и ее следа:

$$
\begin{gathered}
R(x, y)=\int_{0}^{\infty}\left(\frac{2 \pi \operatorname{sh}(2 \omega t)}{m \omega}\right)^{-D / 2} \exp \left[\frac{m \omega\left(2 x y-\operatorname{ch}(\omega t)\left(x^{2}+y^{2}\right)\right)}{2 \operatorname{sh}(\omega t)}+\lambda t\right] d t \\
\operatorname{Tr} R(\lambda)=\int_{0}^{\infty}\left(2 \operatorname{sh}\left(\frac{\omega t}{2}\right)\right)^{-D} e^{t \lambda} d t
\end{gathered}
$$

В рассматриваемом случае спектр

$$
\lambda_{n}=\omega\left(n+\frac{D}{2}\right)
$$

совпадает с известным спектром гармонического осциллятора при целых $D$.

3.4.2. Случай вещественного параметра с. Оператор дилатации $H$ антиэрмитов, поэтому при вещественном параметре $c$ гамильтониан $\mathcal{H}(a, b, c)$ и его резольвента неэрмитовы. Все приведенные выше формулы для резольвенты и ее следа выполняются. Нетрудно также убедиться, что

$$
R_{\lambda}(x, y)=R_{\lambda}^{*}(x, y)=\left.R_{\lambda}(y, x)\right|_{c \rightarrow-c} .
$$

При целом $D$ собственные функции резольвенты в проекторе $P_{n}(x, y)(18)$ вещественны и $\bar{\psi}_{k}^{(n)}(x)=\left.\psi_{k}^{(n)}(x)\right|_{c \rightarrow-c}$.

3.4.3. Случай мнимого параметра $c$. При мнимом параметре $c$ оператор $\mathcal{H}(a, b, c)$ становится эрмитовым. Параметр $f$ может быть положительным, равным нулю или мнимым. Если $f>0$, то, как и в случае вещественного $c$, резольвента и ее спектр имеют вид (15), (17). Для резольвенты и ее собственных функций в (18) выполняются соотношения

$$
R_{\lambda}(x, y)=R_{\lambda}^{*}(y, x), \quad \bar{\psi}_{k}^{(n)}(x)=\psi_{k}^{(n) *}(x) .
$$


При $f=0$ из (15) получаем интегральное представление для резольвенты

$$
R_{\lambda}(x, y)=\int_{0}^{\infty}(4 \pi a t)^{-D / 2} \exp \left[\frac{c t\left(x^{2}-y^{2}\right)-(x-y)^{2}}{4 a t}+t \lambda\right] d t .
$$

Полученный результат можно выразить через цилиндрическую функцию мнимого аргумента [15]:

$$
\begin{aligned}
& K_{\nu}(z)= \frac{1}{2}\left(\frac{z}{2}\right)^{\nu} \int_{0}^{\infty} \frac{e^{-t-z^{2} / 4 t}}{t^{-\nu-1}} d t \\
& R_{\lambda}(x, y)=2(4 \pi a)^{-D / 2} e^{c\left(x^{2}-y^{2}\right) / 4 a}\left(-\frac{(x-y)^{2}}{4 a \lambda}\right)^{(D-2) / 4} \times \\
& \\
& \times K_{(D / 2-1)}\left(\sqrt{-(x-y)^{2} \frac{\lambda}{a}}\right)
\end{aligned}
$$

Выражение (19) отличается лишь множителем $e^{c\left(x^{2}-y^{2}\right) / 4 a}$ от резольвенты свободной частицы массы $m=1 / 2 a$, поэтому спектр резольвенты при $f=0$ непрерывный. Если $f$ стремится к нулю, оставаясь положительным, то расстояние между точками спектра (17) также стремится к нулю и в пределе он заполняет всю полуось $\lambda \geqslant 0$.

При $f=0$ след резольвенты вычисляется явно:

$$
\operatorname{Tr} R_{\lambda}=\int_{0}^{\infty}(4 \pi a t)^{-D / 2} e^{t \lambda} d t=\left(-\frac{\lambda}{4 \pi a}\right)^{D / 2-1} \Gamma\left(1-\frac{D}{2}\right) .
$$

В случае мнимого $f=i g$ мы можем получить интегральные представления для резольвенты и ее следа из (15), (16), выбрав в этих выражениях $f=\epsilon+i g$, где $g$ вещественно, $\epsilon>0$, и после вычисления интегралов, положив $\epsilon=0$ :

$$
\begin{aligned}
R_{\lambda}(x, y) & =\lim _{\epsilon \rightarrow+0} \int_{0}^{\infty}\left(4 \pi a \frac{\sin \left(g_{\epsilon} t\right)}{g_{\epsilon}}\right)^{-D / 2} e^{G\left(x, y, a, c, g_{\epsilon}\right)+t \lambda} d t, \\
G(x, y, a, c, g) & =\frac{c \sin (g t)\left(x^{2}-y^{2}\right)-g \cos (g t)\left(x^{2}+y^{2}\right)+2 g x y}{4 a \sin (g t)}, \\
\operatorname{Tr} R(\lambda) & =\lim _{\epsilon \rightarrow+0} \int_{0}^{\infty}\left(2 \sin \frac{g_{\epsilon} t}{2}\right)^{-D} e^{t \lambda} d t, \quad g_{\epsilon}=g-i \epsilon .
\end{aligned}
$$

В рассмариваемом случае спектр резольвенты $\lambda_{n}=i g(n+D / 2)$ дискретный, чисто мнимый. При $g>0$ он находится на положительной части мнимой оси, а при $g<0-$ на отрицательной.

Для резольвенты выполняются соотношения

$$
R_{\lambda}(x, y)=\left.R_{\lambda}(y, x)\right|_{c \rightarrow-c}, \quad R_{\lambda}^{*}(x, y)=\sum_{n=0}^{\infty} \frac{P_{n}^{*}(x, y)}{\lambda_{n}^{*}-\lambda} .
$$

Таким образом, спектр $\lambda_{n}^{*}=-i g(n+D / 2)$ оператора $R_{\lambda}^{*}(x, y)$ не совпадает со спектром $R_{\lambda}(x, y)$, и при целочисленном $D$ в выражении (18) для проектора $P_{n}(x, y)$ мы имеем $\bar{\psi}_{k}^{(n)}(x)=\left.\psi_{k}^{(n)}(x)\right|_{c \rightarrow-c}$.

В случае мнимого параметра $c$ гамильтониан $\mathcal{H}(a, b, c)$ является самосопряженным в пространстве $L_{2}$, но его собственные функции пространству $L_{2}$ не принадлежат. Этим объясняются свойства оператора резольвенты и ее спектра при мнимом $f$. 
Заметим также, что полученные нами выражения для резольвенты и ее спектра при мнимом $f$ применимы при $a>0, b<0, c=0$ для описания взаимодействия частицы с отталкивающим центром.

\section{4. ЗАКЛЮЧЕНИЕ}

Мы провели расчеты резольвенты обобщенного изотропного осциллятора, используя коммутационные соотношения алгебры $s l(2)$ операторов $p^{2}, q^{2}, H$. Для резольвенты и ее следа получены интегральные представления, аналитические свойства которых исследованы при различных значениях параметров модели. Методы вычисления не зависят явно от выбора размерности пространства $D$, поэтому $D$ можно считать произвольным параметром, для которого допустимы не только целочисленные значения. Мы надеемся, что использованные в нашей работе методы окажутся эффективными и для более сложных изотропных моделей.

Благодарности. Работа выполнена при поддержке Санкт-Петербургского государственного университета (грант № 11.38.660.2013).

\section{Список литературы}

[1] D. Kreimer, Phys. Rep., 363:4-6 (2002), 387-424, arXiv: hep-th/0010059; D. Kreimer, Knots and Feynman Diagrams, Cambridge Lecture Notes in Physics, 13, Cambridge Univ. Press, Cambridge, 2000.

[2] S. Moch, P. Uwer, S. Weinzierl, J. Math. Phys., 43:6 (2002), 3363-3386, arXiv: hep-ph/0110083.

[3] A. I. Davydychev, R. Delbourgo, J. Math. Phys., 39:9 (1998), 4299-4334, arXiv: hep-th/9709216.

[4] D. J. Broadhurst, Eur. Phys. J. C, 8:2 (1999), 311-333.

[5] A. I. Davydychev, M. Yu. Kalmykov, Nucl. Phys. B, 605:1-3 (2001), 266-318.

[6] V.A. Smirnov, Nucl. Phys. B: Proc. Suppl., 116 (2003), 417-421, arXiv: hep-ph/0209295; Phys. Lett. B, 547:3-4 (2002), 239-244, arXiv: hep-ph/0209193; 500:3-4 (2001), 330-337, arXiv: hep-ph/0011056; 460:3-4 (1999), 397-404.

[7] А.Н. Васильев, Квантовополевая ренормгруппа в теории критического поведения и стохастической динамике, Изд-во ПИЯФ, СПб., 1998.

[8] F. V. Tkachov, Phys. Lett. B, 100:1 (1981), 65-68; K. G. Chetyrkin, F. V. Tkachov, Nucl. Phys. B, 192:1 (1981), 159-204.

[9] А. Н. Васильев, Ю. М. Письмак, Ю. Р. Хонконен, ТМФ, 47:3 (1981), 291-306.

[10] A. Zamolodchikov, Phys. Lett. B, 97:1 (1980), 63-66.

[11] A. P. Isaev, Nucl. Phys. B, 662:3 (2003), 461-475, arXiv: hep-th/0303056.

[12] Э. Ч. Титчмарш, Разложение по собственным функциям, связанные с дифберенциальными уравнениями второго порядка, 1 часть, ИЛ, М., 1960.

[13] В. Л. Бахрах, С. И. Ветчинкин, ТМФ, 6:3 (1971), 392-402.

[14] В. Л. Бахрах, С. И. Ветчинкин, С. В. Христенко, ТМФ, 12:2 (1972), 223-226.

[15] И. С. Градштейн, И.М. Рыжик, Таблицы интегралов, сумм, рядов и произведений, Физматгиз, М., 1963. 